\title{
Bilateral lumbar Ultrasound-guided Erector Spinae Plane Block versus Local Anaesthetic Infiltration for Perioperative Analgesia in Lumbar Spine Surgery: A Randomized Controlled Trial
}

\author{
Alessandro Vergari \\ IRCCS Fondazione Policlinico Universitario A. Gemelli \\ Luciano Frassanito (D lucfras75@hotmail.com ) \\ IRCCS Fondazione Policlinico Universitario A. Gemelli \\ Mariangela Muro \\ IRCCS Fondazione Policlinico Universitario A. Gemelli \\ Roberta Nestorini \\ IRCCS Fondazione Policlinico Universitario A. Gemelli \\ Angelo Chierichini \\ IRCCS Fondazione Policlinico Universitario A. Gemelli \\ Marco Rossi \\ IRCCS Fondazione Policlinico Universitario A. Gemelli

\section{Enrico Stasio} \\ Catholic University of the Sacred Heart
}

\section{Research Article}

Keywords: spine surgery, postoperative pain, erector spine pain block, multimodal analgesia

Posted Date: May 10th, 2021

DOI: https://doi.org/10.21203/rs.3.rs-464372/v1

License: (c) (i) This work is licensed under a Creative Commons Attribution 4.0 International License.

Read Full License

Version of Record: A version of this preprint was published at Minerva Anestesiologica on February 1st, 2022. See the published version at https://doi.org/10.23736/S0375-9393.22.15950-X. 


\section{Abstract}

\section{Background}

Lumbar spinal surgery is associated with severe postoperative pain. We examined the analgesic efficacy of bilateral lumbar ultrasound-guided erector spinae plane block (ESPB) with ropivacaine compared with local infiltration of ropivacaine.

\section{Methods}

Twenty-four patients undergoing elective lumbar arthrodesis were randomly divided into two groups. Control group received $0.375 \%$ ropivacaine $40 \mathrm{ml}$ through the wound, and ESPB group received preoperative bilateral ESPB with $0.375 \%$ ropivacaine $40 \mathrm{ml}$. The primary outcome was postoperative pain intensity at rest using a Numeric Rating Scale (NRS). Secondary outcomes included difference in pain intensity between pre-intervention and defined timepoints, total amount of opioid analgesic requested by the patients at the same timepoints, the incidence of any adverse event, and the length of hospital stay (LOS) after surgery.

Results

After surgery we detected a NRS value of $1.9 \pm 1.6$ in ESPB group and $6.0 \pm 1.7$ in Control group $(p<0.05)$. In the ESPB group we found a significant decrease (from $6.3 \pm 1.6$ to $1.9 \pm 1.7$ ) of NRS score after surgery compared to pre-surgery values. About the opioid consumption we found a total sufentanil tablets consumption of $17 \pm 9$ and $10 \pm 2$ at $48 \mathrm{~h}$ for Control group and ESPB group, respectively. Concerning LOS all patients in the Control group and 9 of the ESPB group were discharged after 72 hours; 3 patients in the ESPB group left the ward after 48 hours.

\section{Conclusion}

Bilateral ultrasound-guided ESPB offers improved postoperative analgesia compared with local infiltration in patients undergoing lumbar spinal surgery.

Trial Registration Number on Clinicaltrial.gov: NCT04123106

\section{Introduction}

Patients undergoing spine surgery frequently suffer from postoperative pain [1]. A large retrospective study showed that after a wide range of surgical procedures performed in several hospitals in Germany, postoperative pain was most severe among the patients undergoing spinal fusion surgery [2]. Pain can also be a source of considerable preoperative distress, and in many of these patients the onset of a real chronic pain affecting long-term outcomes has been recognized [3,4]. Severe postoperative pain reduces patients' satisfaction and can delay postoperative recovery and discharge from the hospital $[5,6]$. An adequate control of postoperative pain after spine surgery is critical and leads to reduced postoperative 
complications and length of hospital stay [1-6]. The recent work from the PROSPECT groups tried to identify the analgesic regimen for optimal pain management after spine surgery, recommending multimodal pain management [7-9]. More recently, pathways of care for spine surgery which emphasize opioid-sparing local anesthetic-based regional techniques have been described [1,7,8,10-13]. A regional technique suitable for spine surgery should cover the innervation of the relevant vertebrae and paravertebral muscles and include the dorsal roots of the spinal nerves at the level of surgery [14]. Wound infusion with local anesthetic (LA) agents can attenuate pain blocking peripheral nerve endings for several hours after operation [12,13]. Erector spinae plane block (ESPB) is a novel regional anesthesia technique in which LA are injected into the plane between the fascia of the erector spinae muscle and the vertebral transverse process, involving the dorsal rami of spinal nerves, and is safe and easy to perform under ultrasound (US) guidance $[15,16]$. The ESPB seems to be a promising technique as a part of multimodal anesthetic regimen to manage postoperative pain after spine surgery $[17,18]$.

The aim of this randomized controlled trial is to compare the analgesic efficacy of preoperative bilateral US guided lumbar ESPB with preoperative LA wound infiltration after lumbar spine surgery.

\section{Methods}

This is a prospective, randomized, parallel-group, trial performed in one hospital. The research approach was approved by the internal Ethics Committee (ID 2982, 6 February 2020) and registered on clinicaltrial.gov (10/10/2019, registration number NCT04123106).

Adult patients planned to receive surgical treatment of elective open lumbar arthrodesis at department of vertebral surgery of IRCCS Policlinico A. Gemelli Foundation of Rome, from May 2020 to September 2020 were enrolled in the study. Inclusion criteria: 1 ) age of 18-75 years old, no gender preference; 2) patients suffering from intractable lumbar pain, with or without lower limbs radiation, walking limitation (neurogenic claudication) and a different degree of daily life activity limitation, due to degenerative or isthmic spondylolisthesis, lumbar stenosis and/or spinal deformities; 3 ) surgical treatment of vertebral arthrodesis and decompression of neurologic structures (when required). Exclusion criteria: 1) opioid tolerance (preoperative treatment with opioids for more than 30 days and/or a morphine dosage $>15 \mathrm{mg}$, or morphine equivalent, in the three months before surgery); 2) documented sleep apnoea or home oxygen therapy; 3 ) history of alcohol or drug abuse; 4) allergy or hypersensitivity to opioids or LA; refusal of the patient. Written informed consent was conventionally signed by all patients the day before surgery.

The patients were randomized to receive erector spine plane block (ESPB group) or wound infiltration (Control group) using a computer-generated randomization table. Patients were blinded to the treatment assignment.

\section{Anesthetic procedure and interventions}


All the patients enrolled were managed with open lumbar vertebral arthrodesis with decompression of neurologic structures (when required) through a standard posterior midline approach.

General anesthesia was induced with propofol plus sufentanil, and rocuronium was administered to facilitate tracheal intubation. Anesthesia was maintained using propofol and sufentanil titrated to achieve hemodynamic stability and adequate anesthetic depth, with a Bispectral Index (BIS ${ }^{T M}$, Covidien/Medtronic, Minneapolis, USA) values between 40 and 60 . Once placed in the prone position, in the ESPB group, with aseptic technique, a low-frequency ultrasound probe (M-turbo, Sonosite, Fujifilm, Japan) in a sterile sleeve was used to identify the desired vertebral lamina by paramedian sagittal scan starting from the sacral regions. Then the probe was moved $2-3 \mathrm{~cm}$ lateral to the midline to identify the tip of the corresponding transverse process. After LA infiltration, a 21-gauge $100 \mathrm{~mm}$ needle (Ultraplex 360, B Braun, Melsungen, Germany) was inserted in-plane from cranial to caudal direction until it contacted the corresponding transverse process. After verifying the placement of the needle tip by hydrodissection of the interfascial plane with $2 \mathrm{~mL}$ normal saline, $20 \mathrm{~mL} 0.375 \%$ ropivacaine was injected. The same procedure was repeated on the contralateral side.

In the control group, wound infiltration with ropivacaine $0.375 \% 40 \mathrm{~mL}$ was performed by surgeons at the surgical site at the end of surgery.

Motor-evoked potentials and freerunning electromyography were performed during surgery.

After the surgery all patients received an around-the-clock regimen of ketoprofen and acetaminophen and self-administered sufentanil tablets [SSTs (Zalviso®, Grunenthal, Germany)] as needed [19].

\section{Outcome evaluation}

The primary measure was pain intensity at Recovery Room (RR) arrival (time zero), assessed through a 11-point Numerical Rating Scale (NRS 0: no pain, 10: the worst pain imaginable). Patients had to be cooperative, oriented and tranquil (Ramsay Sedation Scale Score $=2$ ) at the time of assessment. The evaluations were made by an anaesthesiologist blinded to the group allocation of the patient.

Secondary outcomes were: 1) difference in pain intensity between pre-intervention and defined timepoints (RR arrival, at 15 minutes, 30 minutes, 60 minutes, 2, 6, 12, 24, 48 hours); 2) total amount of SSTs requested at the same timepoints; 3 ) number of any adverse event (sickness, vomiting, itching, numbness, respiratory side effects, procedure-related complications); 4) the length of hospital stay (LOS) after surgery.

\section{Statistical Analysis}

Statistical analysis was performed by using the Statistical Package for Social Science (SPSS), release 23.0. Continuous variables were expressed as mean \pm standard deviation [SD (range)], categorical 
variables displayed as frequencies and the Mann-Whitney and Kruskal-Wallis ANOVA nonparametric tests were used to compare different groups at different timings and timing differences within subjects, respectively. Categorical variables were compared using the chi square test. For sample size calculation we used data from a pilot study on 3 controls and patients; a reduction of NRS score at RR timing of at least 2 points in the ESPB in respect to control subgroup was defined as the primary endpoint. A sample size of 12 subjects in each group was estimated for $90 \%$ power and alfa of 0.05 . A $p$ value of less than 0.05 was considered statistically significant.

\section{Results}

Of 35 consecutive patients scheduled for lumbar surgery from May 2020 to September 2020, 24 met the inclusion criteria and were enrolled in the study (the detail of CONSORT diagram for this study is found in Figure 1). Ultimately, there were 12 patients in the ESPB group and 12 patients in the Control group for final analysis. There was no significant difference between groups in demographics data (gender, age, BMI, $p>0.05$ ), as shown in Table 1. Also, similar NRS levels in the two groups are observed before surgery (Figure 2). Duration of surgery was similar in the two groups (mean $250 \pm 51$ min in ESPB group vs $237 \pm 65$ min in Control group). All the patients underwent open lumbar vertebral arthrodesis; 2 patients in Control group and 1 patient in ESPB group were treated also with decompression of neurologic structures.

\section{Table 1}

Demographical characteristic of the population under study. ESPB: Erector Spinae Plane Block.

\begin{tabular}{|llll|}
\hline & $\begin{array}{l}\text { Control group } \\
(\mathbf{n}=12)\end{array}$ & $\begin{array}{l}\text { ESPB group } \\
(\mathbf{n}=12)\end{array}$ & $\begin{array}{l}\text { Significance } \\
(\mathbf{p})\end{array}$ \\
\hline Age $(\mathrm{yrs})$ & $60 \pm 11$ & $58 \pm 10$ & 0.698 \\
\hline Gender (male) & $33 \%$ & $42 \%$ & 0.673 \\
\hline Body Mass Index & $27.4 \pm 3.9$ & $25.4 \pm 2.8$ & 0.153 \\
\hline ASA score [1/2/3/4] (n) & $1 / 11 / 0 / 0$ & $4 / 8 / 0 / 0$ & 0.317 \\
\hline
\end{tabular}

About pain scores at arrival in RR we detected a NRS value of $1.9 \pm 1.6$ in ESPB group and $6.0 \pm 1.7$ in Control group $(p<0.05)$.

Regarding the first of secondary outcomes, we found different timing profile for the two treatments. A timing significant difference (pre-surgery vs 48 h NRS values) can be detected in both groups (Figure 2). 
A different timing profile is revealed for the two treatments: in the Control group no difference of NRS score at arrival in RR compared to pre-surgery values; on the contrary in the ESPB group a significant decrease of NRS score (from $6.3 \pm 1.6$ to $1.9 \pm 1.7$ ) can be observed at the RR determination and confirmed for the entire period of sampling. Significance of each timing difference between Control group and ESPB group are shown in the Figure $2(* p<0.001 ; \# p<0.050)$.

About the sublingual SSTs consumption timing significant difference ( $15 \mathrm{~min}$ vs $48 \mathrm{~h}$ values) can be detected in both groups. A different time increasing rate is observed for the two treatments: a total SSTs of $17 \pm 9$ and $10 \pm 2$ was reached at $48 \mathrm{~h}$ timing for Control group and ESPB group, respectively. Significance of each timing difference between Control group and ESPB group are shown in the Figure 3 $(\# p<0.050)$.

Three patients in ESPB group and 4 in Control group experienced nausea/vomiting, respectively. One patient in ESPB group and 2 in control group suffered dizziness. No complications with regional techniques were reported in the two groups.

Concerning LOS all patients in the Control group and 9 of the ESPB group were discharged after 72 hours; 3 patients in the ESPB group left the ward after 48 hours. Motor-evoked potentials and stimulus-triggered electromyography showed no changes noted from the baseline in both groups.

\section{Discussion}

This randomized controlled study shows that bilateral lumbar US guided ESPB ensures better pain relief compared with wound infiltration with LA after lumbar vertebral arthrodesis.

Lumbar spondylolisthesis, a degenerative disease of the vertebral segmental structure, often hesitates in a displacement of one vertebral body over another with resulting spinal stenosis, neurogenic claudication, radiating pain in the lower extremities, and low-back pain [20]. Surgical treatment of spondylolisthesis may involve a decompression alone or in combination with a fusion procedure [20,21]. Despite advances in treatments and new technologies for spine surgery, large number of patients reported considerable postoperative pain, especially during early postoperative period $[8,21,22]$. Postoperative pain after lumbar spine surgery is related to extensive dissection of the skin, subcutaneous tissues, bones, and ligaments ${ }^{1}$. Moreover, the changes in sagittal alignment are correlated with pain severity [21]. Multimodal pain management including paracetamol and non-steroidal analgesic drugs (NSAIDs) or cicloxygenasys-2 (COX-2) specific inhibitors, the intraoperative use of a low-dose ketamine infusion, and the postoperative use of an epidural infusion of LA are actually recommended for pain control after spine surgery $[7,8]$. As rescue analgesia opioids are still widely used $[7,8]$. Opioids are highly effective on postoperative pain; however, drawbacks of opioids include opioid-induced hyperalgesia and adverse effects such as nausea, gastrointestinal dysmotility, respiratory depression, and risk of opioid habituation [8,9]. More recently, pathways of care for spine surgery which emphasize opioid-sparing LA-based regional techniques have been described $[1,10,11]$. There is growing interest in peripheral pain control by incisional local 
anesthesia: wound infusion with LA can block the peripheral nerve endings and attenuate pain $[1,7,8,12,13]$. Several studies showed that continuous wound infusion seems to guarantee better pain control than intravenous opioids injection, but the patients needed to be inserted with catheters connected to the infusion pump within the wounds, raising the risk of impaired wound healing and local infection [12,13]. Kraiwattanapong et al suggested that intraoperative wound infiltration with multimodal drugs (an opioid, a NSAID, a long-acting LA, and epinephrine) reduced postoperative morphine consumption and decreased pain score after spine surgery; however, a systemic effect of drugs administered into the tissues cannot be excluded [22]. Spine surgery necessitates a midline incision posteriorly, therefore the dorsal rami of the spinal nerves, which innervate the spine and paravertebral tissues, are the nerves of interest [22]. The mechanism of action of ESPB probably is due to the involvement of the dorsal rami of spinal nerves (penetration of LA among muscles), and also to the spread of LA to ventral branches of spinal nerves $[15,18,23,24]$. At lumbar level the erector spinae muscles are larger and thicker than thoracic level, they have tendinous attachments to the lumbar transverse processes, there is the psoas muscle (adherent to the vertebral bodies and to the transverse processes), and there is not a real paravertebral space such as thoracic spine [23,25]. Lumbar nerve roots emerge from the intervertebral foramina in proximity to the anterior surface of the transverse processes and split into dorsal and ventral rami [14]. The dorsal rami penetrate posteriorly into the erector spinae muscle (such as in the thoracic spine), whereas the ventral rami run anteriorly into the psoas muscle and form the lumbar plexus [14]. The fatty intramuscular compartments provide a potential route for injectate spread following a lumbar ESP block [25].

In order to monitor spinal cord efferent pathways during spinal surgery and to avoid side effects after resetting the sagittal body balance, motor evoked potentials in lower limbs generated by transcranial electric stimulation is considered the standard technique [26]. The use of free-running EMG is a simple monitoring technique with surface electrode patches placed in various positions (bilateral vastus medialis, tibialis anterior, gastrocnemius), useful to identify and protect the peripheral nerve structures at risk. Neurotonic burst and train waves, if occurred in response to a surgical manoeuvre, were considered to be abnormal and triggered an alarm to notify the surgeon. The lack of impact on intraoperative electrophysiologic monitoring and the absence of a motor block are additional potential advantages of the ESP block. The limited amount of LA that reaches lumbar ventral rami of spinal nerves and lumbar plexus could explain the absence of motor impairment.

Our results show shorter LOS for patients in ESPB group, although the study is underpowered for this outcome. In our opinion this result is the consequence of better pain control and consequent early mobilization of patients in ESPB group.

Recently Zhang et al in a randomized controlled trial demonstrated that bilateral lumbar ESPB improves postoperative analgesia in patients undergoing lumbar spinal fusion surgery [18]. The authors compared this analgesic technique with a SHAM subcutaneous infiltration with $1 \mathrm{ml}$ of lidocaine [18]. In our opinion the strength of our study is the comparison of ESPB with a certainly effective analgesic technique: this supports the effectiveness of ESPB as main component of an analgesic plan after spine surgery. 
This study has several limitations. First, we did not assess sensory loss in the ESPB group because we performed the block after induction of general anesthesia. Second, the multimodal analgesia regimen theoretically could have affected the results, thinning the differences in postoperative pain scores in the two groups. Third, this is an experience of a single institution and a single orthopaedic team, therefore it may not be representative of patients in other healthcare settings. Further wide comparative controlled randomized studies are needed to validate our findings.

\section{Conclusion}

Bilateral lumbar US-guided ESPB ensures better postoperative pain control and lower opioid consumption compared with local infiltration analgesia after lumbar spine surgery. The block is simple and safe, which makes it worthy of interest when compared with other techniques. Further research is needed to confirm our findings in terms of quality of recovery and rehabilitation.

\section{Declarations}

\section{Ethics approval and consent to participate:}

all the procedures performed in this study were in accordance with the ethical standards of the institutional research committee and with the 1964 Helsinki Declaration and its later amendments. The study was approved by the Ethics Committee of the Medical University of IRCCS Fondazione Policlinico Agostino Gemelli (No. ID 2982, 6 February 2020). All patients enrolled gave written informed consent.

\section{Availability of data and material:}

The datasets used and/or analysed during the current study are available from the corresponding author on reasonable request.

\section{Conflicts of interest/Competing interest:}

The authors have no relevant financial or non-financial interests to disclose. The authors have no conflicts of interest to declare that are relevant to the content of this article. All authors certify that they have no affiliations with or involvement in any organization or entity with any financial interest or nonfinancial interest in the subject matter or materials discussed in this manuscript. The authors have no financial or proprietary interests in any material discussed in this article.

\section{Funding:}

The authors did not receive support from any organization for the submitted work. No funding was received to assist with the preparation of this manuscript. No funding was received for conducting this 
study. No funds, grants, or other support was received.

\section{AUTHOR'S CONTRIBUTION}

A.V. helped design the study, recruit patients, collect the data, draft the manuscript, and review the final manuscript.

L.F. helped design the study, recruit patients, collect the data, draft the manuscript, and review the final manuscript.

M.D.M. recruit patients, collect the data, draft the manuscript, and review the final manuscript.

R.N. recruit patients, collect the data, draft the manuscript, and review the final manuscript.

A.C. recruit patients, collect the data, draft the manuscript, and review the final manuscript.

M.R. helped design the study, analyse data, and review the final manuscript.

E.D.S. helped design the study, analyse data, and review the final manuscript.

\section{ACKNOWLEDGEMENTS}

Not applicable.

\section{References}

1. Mathiesen O, Dahl B, Thomsen BA, Kitter B, Sonne N, Dahl JB, Kehlet H. A comprehensive multimodal pain treatment reduces opioid consumption after multilevel spine surgery. Eur Spine $\mathrm{J}$. Sep;22(9):2089-96.

2. Gerbershagen HJ, Aduckathil S, van Wijck AJ, Peelen LM, Kalkman CJ, Meissner W. Pain intensity on the first day after surgery: a prospective cohort study comparing 179 surgical procedures. Anesthesiology. Apr;118(4):934-44.

3. Nielsen RV, Fomsgaard JS, Dahl JB, Mathiesen O. Insufficient pain management after spine surgery. Dan Med J. May;61(5):A4835.

4. Kehlet $\mathrm{H}$, Jensen TS, Woolf CJ. Persistent postsurgical pain: risk factors and prevention. Lancet. May 13;367(9522):1618-25.

5. Fletcher D, Stamer UM, Pogatzki-Zahn E, Zaslansky R, Tanase NV, Perruchoud C, et al; euCPSP group for the Clinical Trial Network group of the European Society of Anaesthesiology. Chronic postsurgical pain in Europe: An observational study. Eur J Anaesthesiol. Oct;32(10):725-34.

6. DeVine J, Norvell DC, Ecker E, Fourney DR, Vaccaro A, Wang J, et al. Evaluating the correlation and responsiveness of patient-reported pain with function and quality-of-life outcomes after spine 
surgery. Spine (Phila Pa 1976). Oct 1;36(21 Suppl):S69-74.

7. Peene L, Le Cacheux P, Sauter AR, Joshi GP, Beloeil H; PROSPECT Working Group Collaborators; European Society of Regional Anaesthesia. Pain management after laminectomy: a systematic review and procedure-specific post-operative pain management (prospect) recommendations. Eur Spine J. Nov 27.

8. Waelkens, P, Alsabbagh, E, Sauter A, Joshi GP, Beloeil H on behalf of the PROSPECT Working group** of the European Society of Regional Anaesthesia and Pain therapy (ESRA). Pain management after complex spine surgery, European Journal of Anaesthesiology: January 15, Volume Publish Ahead of Print - Issue - doi: 10.1097/EJA.0000000000001448.

9. Wu MH, Wong $\mathrm{CH}$, Niu CC, Tsai TT, Chen LH, Chen WJ. A comparison of three types of postoperative pain control after posterior lumbar spinal surgery. Spine (Phila Pa 1976). Dec 1;36(25):2224-31.

10. Soffin EM, Vaishnav AS, Wetmore DS, Barber L, Hill P, Gang CH, et al. Design and Implementation of an Enhanced Recovery After Surgery (ERAS) Program for Minimally Invasive Lumbar Decompression Spine Surgery: Initial Experience. Spine (Phila Pa 1976). May 1;44(9):E561-E570.

11. Development of an Enhanced Recovery After Surgery (ERAS) approach for lumbar spinal fusion. $J$ Neurosurg Spine. Apr;26(4):411-418.

12. Li K, Ji C, Luo D, Feng H, Yang K, Xu H. Wound infiltration with ropivacaine as an adjuvant to patientcontrolled analgesia for transforaminal lumbar interbody fusion: a retrospective study. BMC Anesthesiol. 2020 Nov 18;20(1):288.

13. Greze J, Vighetti A, Incagnoli P, Quesada JL, Albaladejo P, Palombi O, et al. Does continuous wound infiltration enhance baseline intravenous multimodal analgesia after posterior spinal fusion surgery? A randomized, double-blinded, placebo-controlled study. Eur Spine J. Mar;26(3):832-839.

14. Saito T, Steinke H, Miyaki T, Nawa S, Umemoto K, Miyakawa K, et al. Analysis of the posterior ramus of the lumbar spinal nerve: the structure of the posterior ramus of the spinal nerve. Anesthesiology. Jan;118(1):88-94.

15. Forero M, Adhikary SD, Lopez H, Tsui C, Chin KJ. The Erector Spinae Plane Block: A Novel Analgesic Technique in Thoracic Neuropathic Pain. Reg Anesth Pain Med. Sep-Oct;41(5):621-7.

16. Huang J, Liu JC. Ultrasound-guided erector spinae plane block for postoperative analgesia: a metaanalysis of randomized controlled trials. BMC Anesthesiol. 2020 Apr 14;20(1):83.

17. Chin KJ, Dinsmore MJ, Lewis S, Chan V. Opioid-sparing multimodal analgesia with bilateral bi-level erector spinae plane blocks in scoliosis surgery: a case report of two patients. Eur Spine J. Dec;29(Suppl 2):138-144.

18. Zhang Q, Wu Y, Ren F, Zhang X, Feng Y. Bilateral ultrasound-guided erector spinae plane block in patients undergoing lumbar spinal fusion: A randomized controlled trial. J Clin Anesth. Feb;68:110090.

19. Vergari A, Cortegiani A, Rispoli M, Coluzzi F, Deni F, Leykin Y, et al. Sufentanil Sublingual Tablet System: from rationale of use to clinical practice. Eur Rev Med Pharmacol Sci. Nov;24(22):1189111899. 
20. Liang HF, Liu SH, Chen ZX, Fei QM. Decompression plus fusion versus decompression alone for degenerative lumbar spondylolisthesis: a systematic review and meta-analysis. Eur Spine J. Dec;26(12):3084-3095.

21. Liang Y, Shi W, Jiang C, Chen Z, Liu F, Feng Z, Jiang X. Clinical outcomes and sagittal alignment of single-level unilateral instrumented transforaminal lumbar interbody fusion with a 4 to 5 -year followup. Eur Spine J. Nov;24(11):2560-6.

22. Kraiwattanapong C, Arnuntasupakul V, Kantawan R, Woratanarat P, Keorochana G, Langsanam N. Effect of Multimodal Drugs Infiltration on Postoperative Pain in Split Laminectomy of Lumbar Spine: A Randomized Controlled Trial. Spine (Phila Pa 1976). Dec 15;45(24):1687-1695.

23. Chin KJ, El-Boghdadly K. Mechanisms of action of the erector spinae plane (ESP) block: a narrative review. Can J Anaesth. 2021 Mar;68(3):387-408.

24. Kendall MC, Alves L, Traill LL, De Oliveira GS. The effect of ultrasound-guided erector spinae plane block on postsurgical pain: a meta-analysis of randomized controlled trials. BMC Anesthesiol. 2020 May 1;20(1):99.

25. Macintosh JE, Bogduk N. 1987 Volvo award in basic science. The morphology of the lumbar erector spinae. Spine (Phila Pa 1976). Sep;12(7):658-68.

26. Lieberman JA, Lyon R, Feiner J, Hu SS, Berven SH. The efficacy of motor evoked potentials in fixed sagittal imbalance deformity correction surgery. Spine (Phila Pa 1976). Jun 1;33(13):E414-24.

\section{Figures}


$\underline{\text { Enrollment }} \quad$ Assessed for eligibility $(\mathrm{n}=35)$

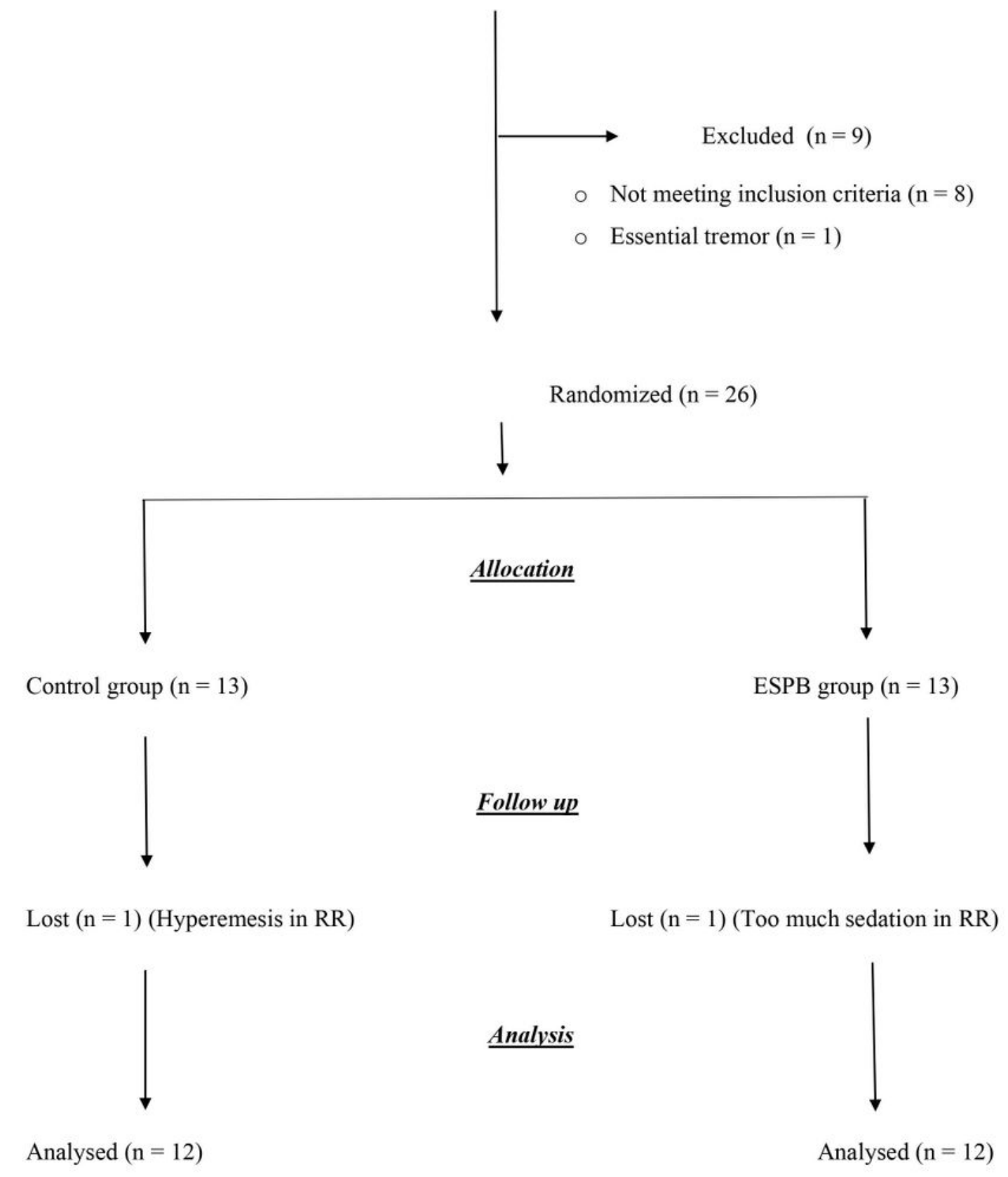

Figure 1

Figure 1

CONSORT Diagram. ESPB: Erector Spinae Plane Block. 


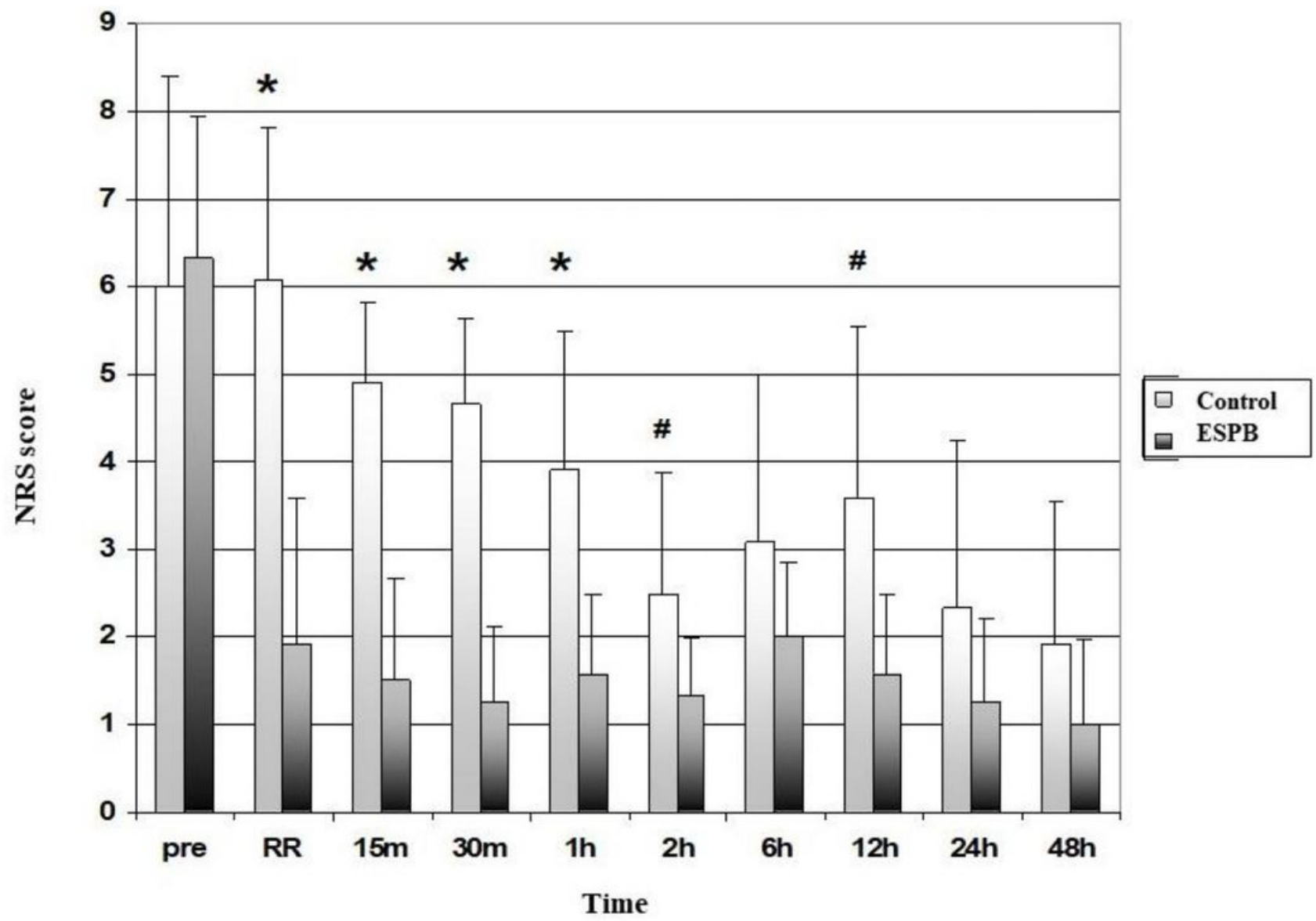

Figure 2

Figure 2

Postoperative pain score as function of timing in the Control group and ESPB group ( $*$ : $p<0.001 ; \#$ : p <0.050). ESPB: Erector Spine Plane Block. NRS: Numerical Rating Scale. 


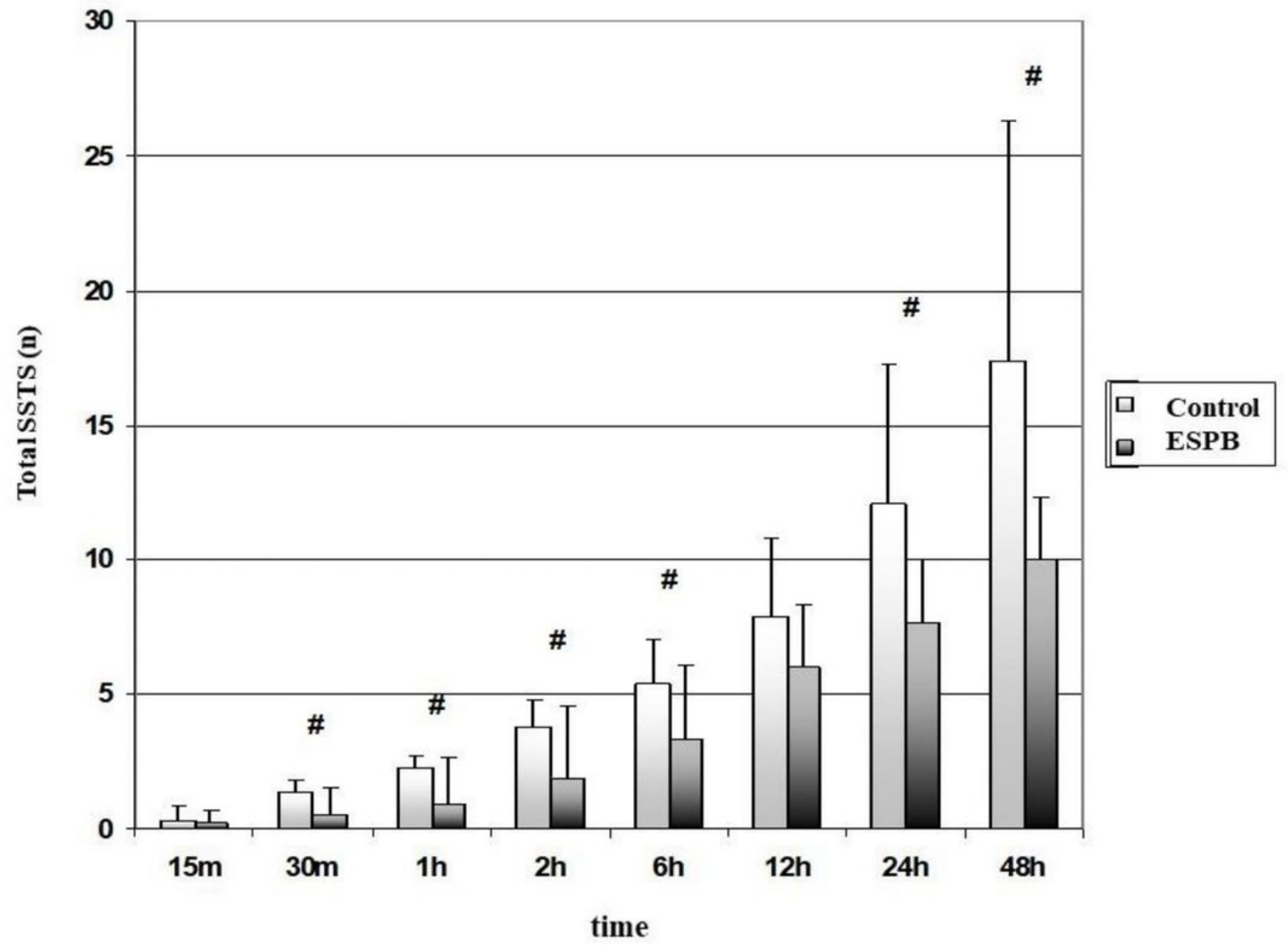

Figure 3

Figure 3

Total number of SSTs used as function of timing in the Control group and ESPB group (\#: $p<0.050)$. SSTS: self-administered sufentanil tablets; ESPB: Erector Spine Plane Block. 